\title{
Conserved expression domains for genes upstream and within the HoxA and HoxD clusters suggests a long-range enhancer existed before cluster duplication
}

\author{
Jessica A. Lehoczky, ${ }^{a}$ Melissa E. Williams, ${ }^{a}$ and Jeffrey W. Innis ${ }^{a, b, *}$ \\ ${ }^{a}$ Department of Human Genetics and \\ ${ }^{\mathrm{b}}$ Department of Pediatrics, Division of Genetics, University of Michigan, Ann Arbor, Ml 48109, USA \\ *Author for correspondence (email: innis@umich.edu)
}

\begin{abstract}
SUMMARY The posterior HoxA and HoxD genes are essential in appendicular development. Studies have demonstrated that a "distal limb enhancer," remotely located upstream of the HoxD complex, is required to drive embryonic autopod expression of the posterior Hox genes as well as the two additional non-Hox genes in the region: Evx2 and Lnp. Our work demonstrates a similar mode of regulation for Hoxa13 and four upstream genes: Evx1, Hibadh, Tax1bp, and Jaz1. These genes all show embryonic (E11.5-E13.5) distal limb and genital bud expression, suggesting the existence of a nearby enhancer influencing the expression of a domain of genes. Comparative sequence analysis between homologous human and mouse genomic sequence upstream of Hoxa13 revealed a remote $2.25-\mathrm{kb}$ conserved noncoding sequence (mmA13CNS) within the fourth intron of the Hibadh gene.
\end{abstract}

mmA13CNS shares a common 131-bp core identity within a conserved noncoding sequence upstream of Hoxd13, which is located within the previously identified distal limb enhancer critical region. To test the function of this conserved sequence, we created mmA13CNS-Hsp86-lacZ transgenic mice. $\mathrm{mmA13CNS}$ directed a wide range of tissue expression, including the central nervous system, developing olfactory tissue, limb, and genital bud. Limb and genital bud expression directed by mmA13CNS is not identical to the patterns exhibited by Hoxa13/Evx1/Hibadh/Tax1bp1/Jaz1, suggesting that $\mathrm{mmA} 13 \mathrm{CNS}$ is not sufficient to fully recapitulate their expression in those tissues. The Evx1- and Evx2-like central nervous system expression observed in these mice suggests that the long-range regulatory element(s) for the Hox cluster existed before the cluster duplication.

\section{INTRODUCTION}

Hox genes encode essential transcription factors for embryonic development of the axial and appendicular body plan of metazoans (McGinnis and Krumlauf 1992; Veraksa et al. 2000). The Hox genes are located in genomic clusters with genes $3^{\prime}$ in the cluster expressed earliest in development and most anterior in the developing axial body plan and genes $5^{\prime}$ in the cluster expressed later in development and more posterior in the organism (Duboule and Morata 1994). Branchiostoma floridae (amphioxus), an extant cephalochordate related to the pre-Hox duplication vertebrate ancestor, has one Hox cluster comprised of 14 genes (Ferrier et al. 2000), yet over vertebrate evolution the genomic clusters duplicated such that mammals have four Hox clusters, HoxA through HoxD, with a total of 39 genes spread among 13 paralogous groups (Ruddle et al. 1994).

Among numerous sites of expression, mammalian development of the appendicular skeleton and external genitalia relies on the $5^{\prime}$ Hox genes of paralog groups A and D (Fro-
mental-Ramain et al. 1996; Mortlock et al. 1996; Kondo et al. 1997; Warot et al. 1997). In the HoxD cluster, genes Hoxd10 through Hoxd13 have been shown to be necessary for proper limb development in mice (Zakany et al. 1997). In the HoxA cluster, Hoxal3 is essential in distal limb development, as shown by genetically engineered Hoxal3 null alleles (Fromental-Ramain et al. 1996; Stadler et al. 2001), the mouse mutant Hypodactyly (Mortlock et al. 1996), and patients with hand-foot-genital syndrome (Mortlock and Innis 1997; Goodman et al. 2000) (OMIM \#140000). During mouse development, Hoxd10 through Hoxd13 are expressed in the mesenchyme of the distal fore- and hindlimb autopods as well as the genital tubercle from mouse embryonic days 10.5 through 13.5 (E10.5-E13.5). This expression is comparable with that of the Hoxd13 paralog, Hoxa13, which shows similar distally restricted limb bud and genital bud expression during embryogenesis.

Investigation into the expression domains of genes $5^{\prime}$ to Hoxd13 or Hoxal3 revealed that additional non-Hox genes share their distal limb and genital bud expression patterns 
during embryonic development in mice. Evx2 and Lunapark (Lnp) are located upstream of Hoxd13, and both have mesenchymal distal limb and genital bud expression as part of their expression domains (Spitz et al. 2003). Evxl is located 5' of Hoxal3 and similarly has distal and genital bud expression during embryonic development (Bastian and Gruss 1990; this study). In addition, Evxl and Evx2 share additional embryonic expression domains in the developing central nervous system where neither $5^{\prime}$ HoxA nor 5' HoxD genes are expressed. The conserved distal limb and genital bud expression domains of the posterior HoxD and $5^{\prime}$ genes are regulated by a global element(s) located further $5^{\prime}$ of $L n p$ (Spitz et al. 2001).

Given that the Hox complexes were derived by duplication of an ancestral group of genes, we hypothesized that a regulatory mechanism similar to that proposed for posterior HoxD genes, which minimally involves enhancers acting over a domain of genes to direct expression in the distal limb and the genital bud, also exists for HoxA. Herein, we examine the spatial and temporal expression pattern of six genes upstream of Hoxal3: Evxl, Hibadh, Tax1bp1, Jaz1, Crebpa, and KIAA0644. We also use upstream HoxA and HoxD sequence comparisons to identify a highly conserved noncoding sequence (CNS) upstream of Hoxal3 and test the function of this element in transgenic lac $Z$ reporter mice.

\section{MATERIALS AND METHODS}

\section{Sequence alignments and analysis}

Human and mouse genomic regions $5^{\prime}$ of HOXA13 and HOXD13 were obtained from the Ensembl Genome Browser (www. ensembl.org). Human DNA sequence was obtained from Build 34, and mouse DNA sequence was obtained from Build 30. Genomic sequences were Repeatmasked (Smit and Green, unpublished results; http://www.repeatmasker.org) and subjected to multiple species alignments using advanced Pipmaker and Multipipmaker with default settings (Schwartz et al. 2000; http://bio.cse.psu.edu/ pipmaker). Conserved noncoding sequences (CNSs) are referred to frequently in this study and where discussed, "central nervous system" is not abbreviated. Sequences homologous to mmA13CNS were obtained by BLAST comparison (Altschul et al. 1990) against NCBI GenBank databases nr, htg, gss, and wgs (www.ncbi.nlm. nih.gov/Genbank).

\section{Transgenic mice and $L a c Z$ staining}

The original Hsp68-lac $Z$ vector (Kothary et al. 1989; DiLeone et al. 1998) was modified to include a NotI-SfiI-NotI cloning site. We obtained this modified vector from Dr. Doug Mortlock (Vanderbilt University, Nashville, TN, USA). mmA13CNS was amplified by polymerase chain reaction from $\mathrm{C} 57 \mathrm{Bl} / 6$ mouse genomic DNA with primers $5^{\prime}$-ATGTGCTCTGCCACTCTCTG and $5^{\prime}$ TGGAATTGGCCTTACGAAAA. The integrity of this element was confirmed by DNA sequencing and subsequently cloned into the NotI site of the Hsp68-lac $Z$ vector. The transgene was linearized, and the vector backbone was removed. Transgene DNA was injected into pronuclei of fertilized B6D2 F2 eggs and implanted into pseudopregnant CD1 females. Embryos were harvested for $\beta$ galactosidase staining at E12.5, and amniotic membranes were collected for transgene genotyping. LacZ staining was performed by standard protocol (Hogan et al. 1994). Embryos used for sections were dehydrated in ethanol/phosphate-buffered saline and subsequently embedded in paraffin by standard protocol. Embryos were sectioned at 6 or $10 \mu \mathrm{m}$, as noted.

\section{In situ hybridization}

Hibadh, Tax1bp1, Crebpa, and Jaz1 cDNAs were amplified from an E12.5 C57Bl/6 mouse limb bud cDNA library and cloned into polymerase chain reaction-4 Topo vector (Invitrogen, Carlsbad, CA, USA). Two clones/probes were made for Hibadh and pooled for in situ hybridization. Hibadh primer pairs were as follows: hibadhex7.F 5'-CAGGCCTTGACCCAAAACTA and hibadh _ $3^{\prime}$ UTR.R 5'-TCATCATTGGCTCTGAAACAG, mm_hibadh.F 5'-GGATTCATTGGACTGGGAAA and mm_hibadh.R 5'-ATTGGGTGTGCTGGCTAAAC. All other primer pairs were as follows: jazl.F 5'-GGAGTCAGACAGTGATGAGTCC and jazl.R 5'-GGTTAAACAATATGCAACATGCC, mmCrebpa.F 5'-TTATCTCAGACCTGGAAGACG and mmCrebpa.R 5'-TTTCCCTAGTGTCCCCACAG, Tax1bplex15.F 5'-GCCCTCAACAAGTCTCAAGG and Tax1bp1_3'UTR.R 5'-CAATCGCCAAGGTGTACAGA. The Evxl clone was kindly provided by Dr. Gail Martin (UCSF, San Francisco, CA, USA). Digoxygeninlabeled anti-sense RNA probes were generated as previously described (Post and Innis 1999). In situ hybridization was carried out as previously described (Bober et al. 1994) except embryos were developed using BM purple substrate (Roche Applied Science, Indianapolis, IN, USA).

\section{RESULTS}

We hypothesized that the mechanism regulating distal limb bud and genital bud expression in posterior HoxD and $5^{\prime}$ genes may have been conserved on the HoxA chromosome. We sought to characterize the embryonic RNA expression patterns of the genes within 1.5 megabases upstream of Hoxa13. Six genes were identified within this interval in human and mouse: Evxl, Hibadh, Taxlbpl, Jazl, Crebpa, and KIAA0644 (Fig. 1). Notably, the HoxA cluster through Crebpa is a conserved syntenic block of genes from at least bony fish through humans, without apparent gene insertions or deletions. It would be of interest to examine gene context upstream of the HoxA clusters in lampreys and sharks; however, this genomic sequence is not available. Evxl, the first gene $5^{\prime}$ of the HoxA cluster, was originally identified to be a homolog of Drosophila even-skipped (Bastian and Gruss 1990). Evxl expression domains are closely correlated to that of Evx2, the first gene upstream of Hoxd13, with expression in the developing neural tube, hindbrain, distal limbs, and genital bud (Bastian and Gruss 1990; Dollé et al. 1994) (Fig. 1, E-H). The expression seen for Evxl in the distal limbs and 


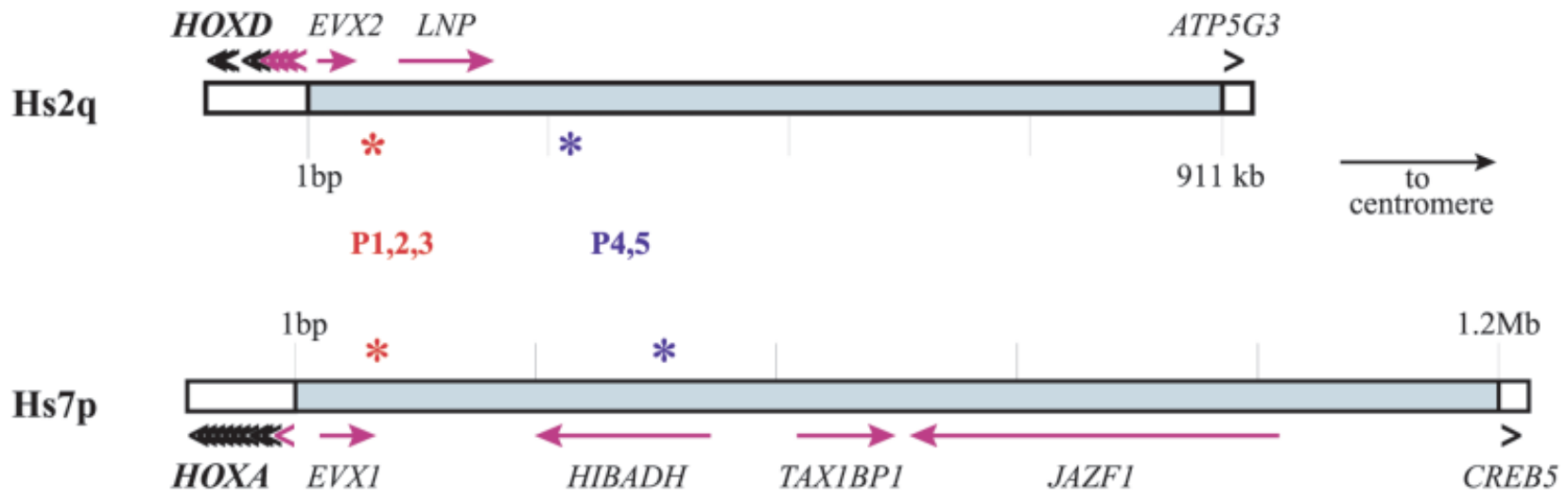

E11.5
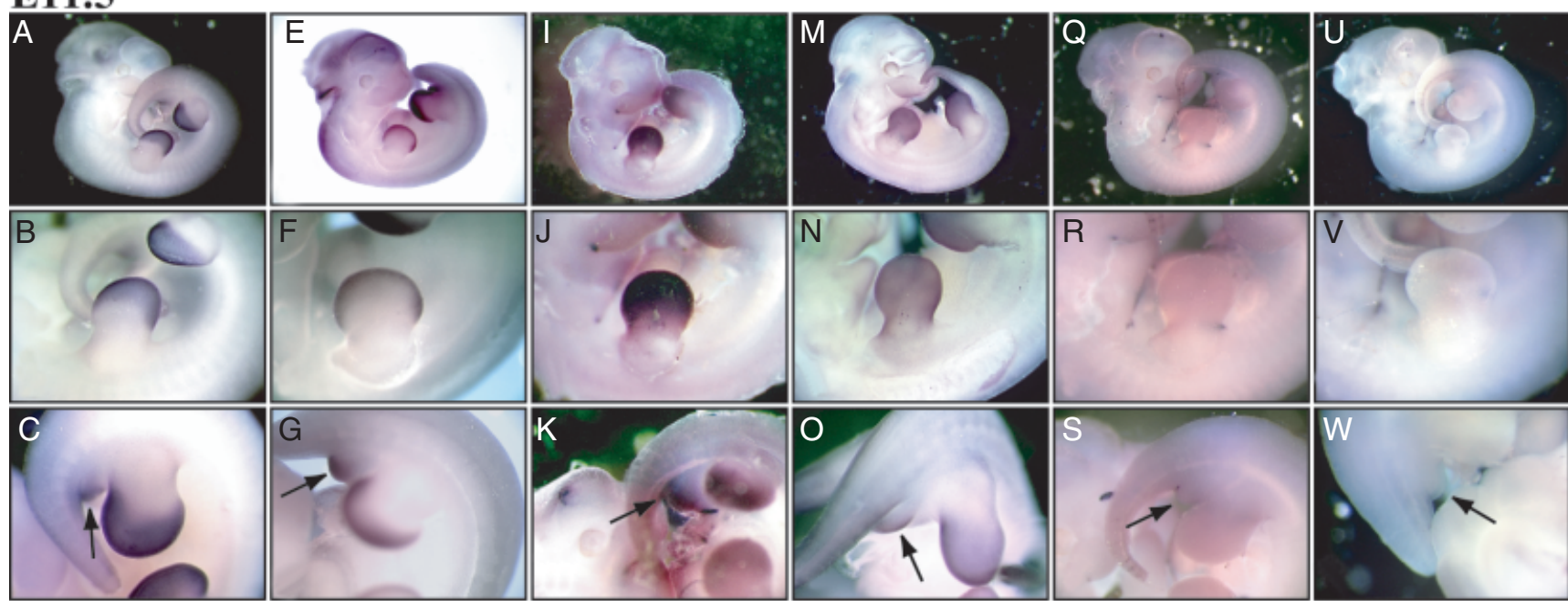

Hoxa13

Evx1

Hibadh

Tax1bp1

Jaz1

Crebpa

E12.5
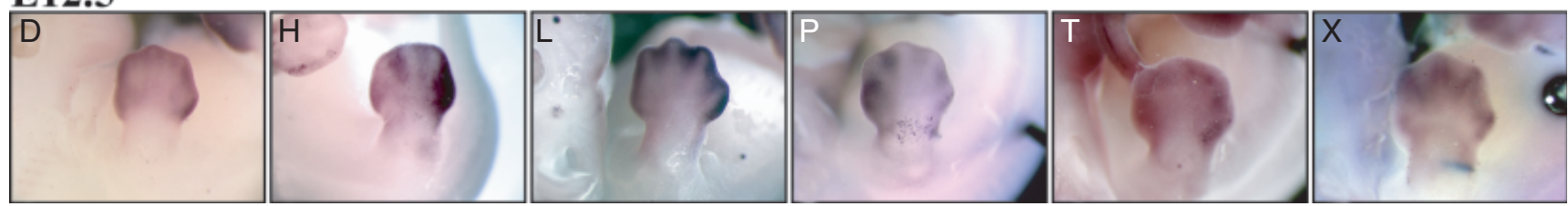

Fig. 1. Genomic organization and expression domains of genes upstream of HoxA. Top shows $911 \mathrm{~kb}$ of human genomic sequence $5^{\prime}$ of $H O X D 13$, where base 1 is the first base before the start of HOXD13 transcription and base 911,179 is the last base before the start of ATP5G3 transcription. Below this diagram 1.2 Mb of human genomic sequence $5^{\prime}$ of $H O X A 13$ is drawn to scale. Base 1 is the first base before the start of HOXA13 transcription and base $1.2 \mathrm{Mb}$ is the first base before the start of CREB5. Arrows below gene names denote the direction of transcription and those in pink share common embryonic distal limb expression patterns. Shaded gray portions represent sequence used for comparative analysis. Red asterisks denote the relative location of paraCNSs 1, 2, and 3. Blue asterisks show the relative location of paraCNS4/5 and A13CNS/D13CNS, respectively. Bottom shows whole-mount RNA expression domains of Hoxal3 (A-D), Evxl (E-H), Hibadh (I-J), Taxlbpl (M-P), Jazl (Q-T), and Crebpa (U-X) for mouse embryos. A, E, I, M, Q, and U show E11.5 right sides, and B, F, J, N, R, and V show close-up of right E11.5 forelimbs. Arrows in C, G, K, O, S, and W show E11.5 genital bud expression. D, I, P, T, and X show E12.5 forelimb expression, and H shows E12.5 hindlimb, as Evxl begins turning off at E12.5 in the forelimb.

genital bud are similar to that of Hoxal3 in terms of spatial and temporal characterization (Fig. 1, A-D).

3-Hydroxyisobutyrate dehydrogenase $(H I B A D H)$ is located $325 \mathrm{~kb} 5^{\prime}$ of human HOXA13. HIBADH is expressed prima- rily in the adult liver and is involved in valine catabolism (Rougraff et al. 1988). There is no previous evidence of embryonic expression for this gene. Hibadh anti-sense RNA in situ hybridization for mouse E10.5 through E13.5 revealed a 
mesenchymal staining pattern highly similar to that of Hoxa13, with mesenchymal distal limb and genital bud staining (Fig. 1, I-J). Taxl binding protein 1 (TAX1BP1), located $540 \mathrm{~kb} 5^{\prime}$ of $H O X A 13$, has been shown in vitro to be a substrate for caspase-3-like proteases in the tumor necrosis factor-induced apoptotic pathway (De Valck et al. 1999). Although no embryonic expression profile has been reported for this gene, RNA in situ hybridization shows a distal limb and genital bud staining pattern similar to that of Hoxal3 (Fig. 1, M-P). Juxtaposed with another zinc-finger gene 1 (Jazl; human, JAZF1), located $630 \mathrm{~kb} 5^{\prime}$ of human $\mathrm{HO}$ $X A 13$, has only been reported in the context of JAZF1/JJAZ fusion protein expression in endometrial stromal neoplasms (Koontz et al. 2001). Evaluation of the developmental RNA expression profile of $\mathrm{Jazl}$ for mouse E10.5 and 11.5 shows low-level, ubiquitous, embryonic expression including the limb and the genital bud (Fig. 1, Q-S). This ubiquitous expression is reproducible and not correlated with time in developing reagent. At E12.5 and E13.5, the expression pattern is dramatically restricted to the interdigital mesenchyme (Fig. 1T), central nervous system, and genital bud (data not shown).

Two additional genes $5^{\prime}$ of Hoxal3 were evaluated for their relative expression during E10.5 through E13.5: Crebpa and KIAA0644. CAMP responsive element binding protein (Crebpa; human, CREB5) shows ventral neural tube, forebrain, and faint autopod staining dissimilar to Hoxal3 and no genital bud expression at E11.5 (Fig. 1, U-W). Crebpa displays an interdigital staining tightly confined to the edges of the condensing mesenchyme at stages E12.5 (Fig. 1X) and E13.5, in addition to forebrain staining (data not shown). KIAA0644, originally isolated from a human brain cDNA library (GenBank accession number AB014544), does not show the characteristic Hoxal3 expression pattern. KIAA0644 expression was observed in the proximal, not distal, limb as well as the forebrain (data not shown).

To identify candidate regulatory elements necessary for the distal limb and genital bud expression pattern observed for Hoxal3 and the four upstream genes, comparative sequence analysis was performed using human and mouse genomic sequence. Comparison of approximately $900 \mathrm{~kb}$ of human genomic sequence $5^{\prime}$ of $H O X D 13$ with $1.2 \mathrm{Mb}$ of human sequence $5^{\prime}$ of $H O X A 13$ yielded five conserved noncoding sequences, and identical results were obtained by comparison with the paralogous mouse regions (Table 1). All five CNSs were core sequence identities, which fell within larger homologous CNSs found between the human and mouse homologous cluster comparison. The five elements were further characterized by their locations. We define a paraCNS as conserved noncoding sequences in common between paralogous Hox clusters. ParaCNS1, paraCNS2, and paraCNS3 are all located immediately (approximately $2 \mathrm{~kb}$ /approximately $4 \mathrm{~kb}$ ) downstream of $E V X 1 / E V X 2$ (Fig. 1, red asterisks) and are within the previously described putative regulatory element R1 (Goodman et al. 2002). ParaCNS4 and paraCNS5 are located within $1 \mathrm{~kb}$ of each other in both the context of $5^{\prime} H O X D 13$ and $5^{\prime} H O X A 13$ but are individually situated over $200 \mathrm{~kb}$ away from $H O X D 13$ and over 350 kb away from HOXA13 (Fig. 1, blue asterisks).

ParaCNS4 and paraCNS5 are core identities of $131 \mathrm{nt} /$ $68 \%$ and $105 \mathrm{nt} / 60 \%$, respectively, yet when these core sequences were compared with homologous sequences of other species, they fell within larger highly conserved sequences. $5^{\prime}$ of $H O X A 13$, paraCNS4 and paraCNS5 fall within the fourth intron of HIBADH embedded within a CNS of approximately $2.25 \mathrm{~kb}$ : A13CNS (Fig. 2). These two paraCNSs also fall within an approximately $3-\mathrm{kb}$ CNS $5^{\prime}$ of HOXD13: D13CNS. Notably, they are otherwise unique in the mouse and human genomes. Interestingly, D13CNS is a candidate conserved sequence within the previously identified critical region for the "distal limb enhancer" (Spitz et al. 2003). The coincidence of the paralogous conservation with the distal limb enhancer critical region makes its paralogous counterpart, A13CNS, an appealing enhancer candidate for global regulation of $H O X A 13$ and $5^{\prime}$ genes.

A13CNS function was directly tested in embryonic development in transgenic mice. Mouse A13CNS (mmA13CNS) was placed in cis with a minimal Hsp68-lac $Z$ construct and used as a transgene for E12.5 founder analysis of the embryos. $L a c Z$ staining was observed in a variety of developing structures (Fig. 3). Staining was seen in the superior neural layer and wall, but not the overlying epithelium, of the midbrain (Fig. 3D). In addition, lac $Z$ staining was observed in the presumptive olfactory tissue (Fig. 3E), hindbrain, pons, medulla, and corpus plexus (Fig. 3F). Staining was observed in the distal mantle region of the lumbosacral spinal cord, midway

Table 1. Characterization of $H o x A / D$ paraCNSs

\begin{tabular}{|c|c|c|c|c|}
\hline Conserved Element & Distance from $\mathrm{HOXD} 13$ & Distance from $\mathrm{HOXA13}$ & Length of Homology & $\%$ Identity \\
\hline paraCNS1 & $16,688 \mathrm{bp}$ & $48,674 \mathrm{bp}$ & 209 bp & 64 \\
\hline paraCNS2 & $17,026 \mathrm{bp}$ & $49,509 \mathrm{bp}$ & $47 \mathrm{bp}$ & 87 \\
\hline paraCNS3 & $19,042 \mathrm{bp}$ & $51,049 \mathrm{bp}$ & $205 \mathrm{bp}$ & 64 \\
\hline paraCNS4 & $238,369 \mathrm{bp}$ & $351,994 \mathrm{bp}$ & $131 \mathrm{bp}$ & 68 \\
\hline paraCNS5 & $239,106 \mathrm{bp}$ & $353,107 \mathrm{bp}$ & $105 \mathrm{bp}$ & 66 \\
\hline
\end{tabular}




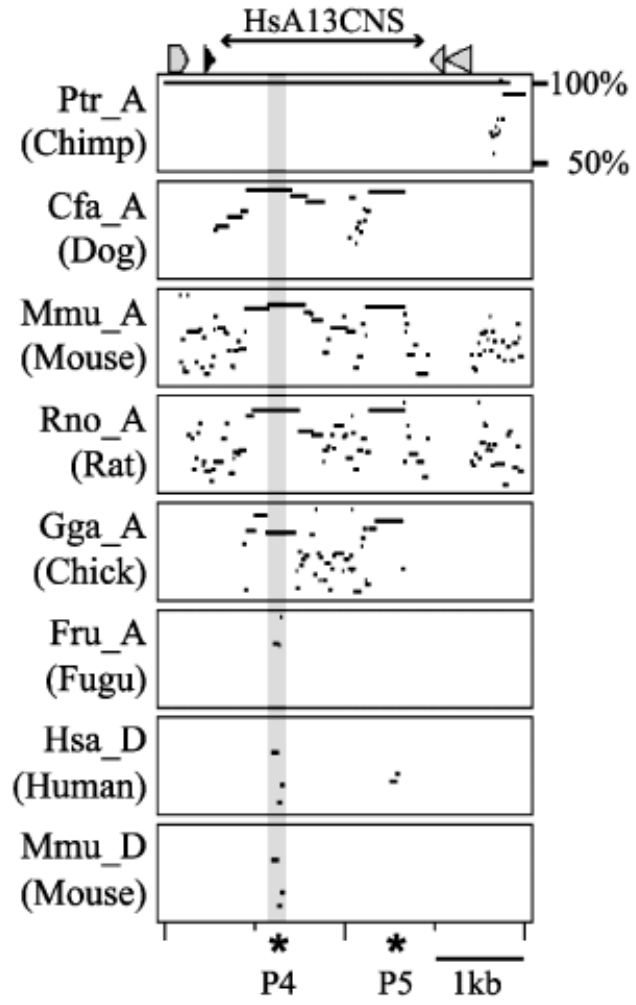

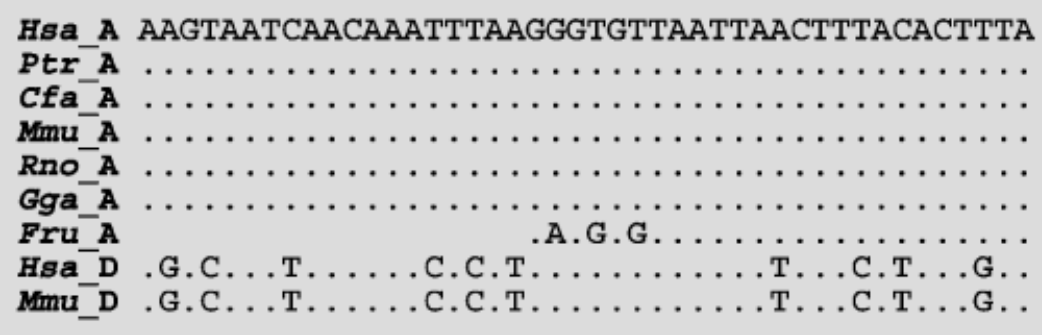

hsa A TCATGTTCAATTAG-TTGTCCTTTCTAGAAAAGATTGCCGTGCA

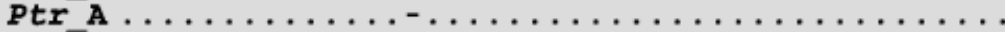

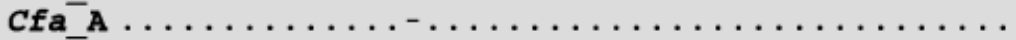

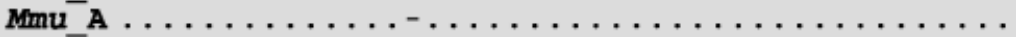

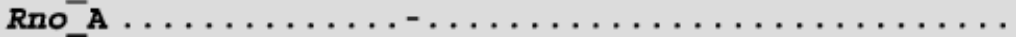

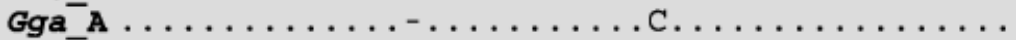
Fru_A ............ . . . C.C. . . . . .

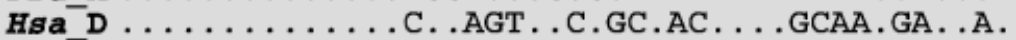
Mmu_D ........... C. AGT . C.GC.AC. . .GC.A.GA. A.

Hsa_A CAATCAGTTTA--GAGCGTGCCATGTTTGATTTGTAAGTGATC

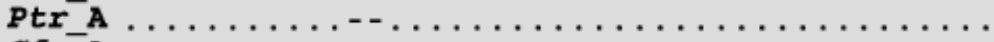

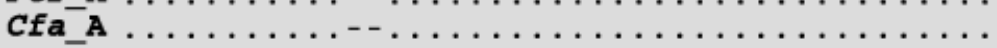

Mmu_A . . . . . . . . . . . . . . . . . . .

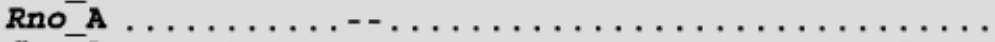

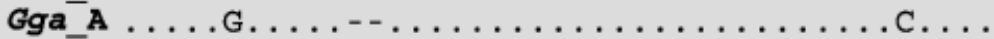

Fru_A.TGCTG.C...AG. . . . . . . . .

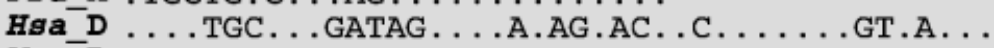

Mmu_D ....TGC...GATAG....A.AG.AC . C.......GT.A...

Fig. 2. Evolutionary conservation of HsA13CNS. Multipipmaker was used to align human A13CNS with six orthologous regions (Hsa, Homo sapiens; Ptr, Pan troglodytes; Cfa, Canis familiaris; Mmu, Mus musculus; Rno, Rattus norvegicus; Gga, Gallus gallus; Fru, Takifugu rubripes) and the human and mouse paralogous regions $5^{\prime}$ of $H O X D 13$. Length of sequence identities versus the human reference sequence are depicted as horizontal lines, and the percent identity from 50 to 100 is measured on the vertical axis. Asterisks represent location of paraCNS4 and paraCNS5. Sequence alignment on the right within vertical gray box is paraCNS4 depicted at the nucleotide level, where $(\cdot)$ represents identity.

between the ventral and dorsal gray horns of the spinal cord (Fig. 3D), and weak staining was visualized in a portion of the mesenchyme of the genital tubercle and the anterior wall of the urogenital sinus (Fig. 3G). Additionally, variable weak staining was observed in the interdigital mesenchyme and proximal limb regions in 5 of 12 expressing founder limbs (Fig. 4, A and B).

Two permanent lines of mice were established for the mmA13CNS-lac $Z$ transgene. Analysis of transgenic embryos from one line at E11.5-E13.5 yielded strong central nervous system staining identical to that of the founders. The neural tube, midbrain, and hindbrain lacZ expression seen in the mmA13CNS mouse is highly similar to the neural expression patterns of Evxl (neural tube and hindbrain) and Evx2 (neural tube, hindbrain, and midbrain) (Dollé et al. 1994) (Fig. 5). Limb staining was observed in proximal limb elements and the presumptive digits at E12.5 and E14.5 (Fig. 4). The lacZ staining in the proximal limb elements at these times (Fig. 4, C-E) may represent HoxA expression in the developing limb musculature (Yamamoto et al. 1998). However, further analysis involving myoblast markers must be used to definitively characterize this lacZ expression pattern, but we hypothesize that a long-range enhancer of posterior HoxA genes in muscle development may lie within mmA13CNS. Profound staining was seen at E13.5 and E14.5 in the olfactory tissue or developing vomeronasal organ (data not shown). Genital mesenchyme lacZ expression that was seen in three of three expressing founders was not seen in any offspring of this permanent line, suggesting a potential negative effect of the transgene insertion site.

In the second permanent line, identical central nervous system expression was observed. Staining in offspring of this line at E11.5 revealed limb and genital bud lacZ staining; however, as with the other line, the precise Hoxal3 expression pattern was not observed. Thus, although timing and general tissue expression may be partly recapitulated with this construct, precise domain expression was not observed. The wide range of reproducible lacZ expression domains confirms the functional capabilities of this highly conserved CNS. The observation of broader reproducible expression in tissues not 


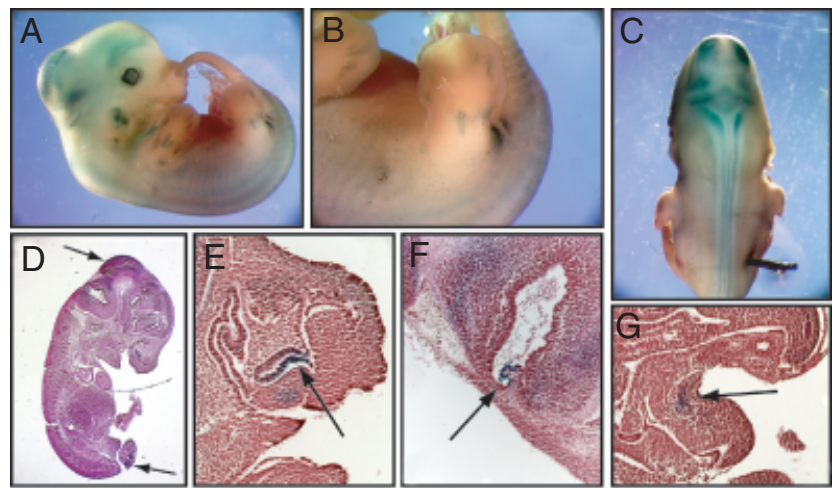

Fig. 3. LacZ expression of mmA13CNS founders at E12.5. Representative $l a c Z$ staining in one of three E12.5 transgenic founders. (A) Wide range of lacZ-positive structures. (B) Whole-mount expression in genital bud in addition to weak limb staining. (C) Central nervous system staining. (D-G) Ten-micrometer paraffin sections of the founder in A-C, with standard hematoxylin and eosin staining. (D) LacZ expression specific to the periphery of the midbrain (top arrow) and caudal neural tube (bottom arrow). (E) Staining specific to the olfactory region. (F) The expression found in the corpus plexus (arrow) and within the hindbrain. (G) Staining in the genital bud mesenchyme.

known to express any of the upstream neighboring genes suggests mmA13CNS may harbor enhancer elements, perhaps for distant genes, capable of directing expression to those tissues. Alternatively, essential negative control elements may be missing. Finally, this $2.25-\mathrm{kb}$ element is not sufficient for full recapitulation of limb bud and genital bud expression for this domain of genes.

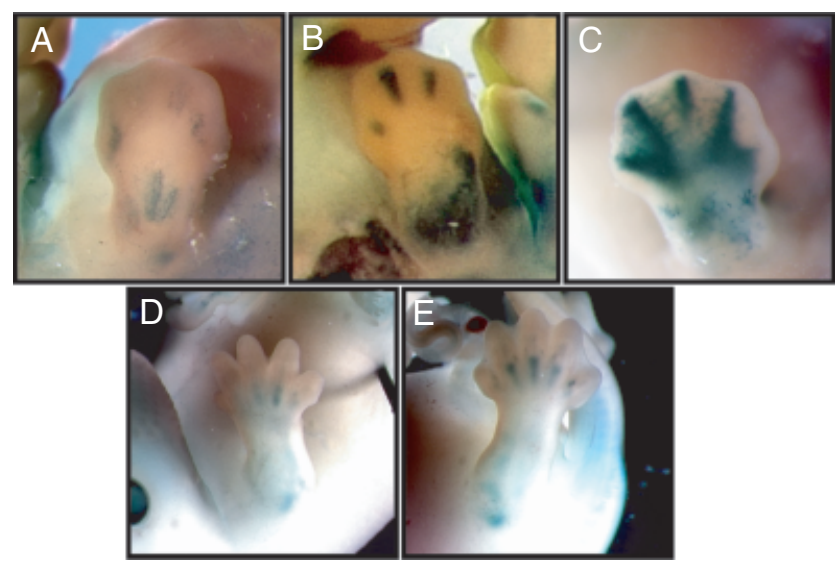

Fig. 4. LacZ expression in mmA13CNS transgenic limbs. LacZstained E12.5 (A-C) and E14.5 (D and E) embryos. (A and B) mmA13CNS transgenic founders. Staining is shown in the interdigital mesenchyme and in proximal limb elements. (C-E) Obtained from the mmA13CNS permanent line. (C) Digital lacZ staining and a diffuse staining in the autopod and the proximal limb. Representative forelimb (D) and hindlimb (E), with distinct digital and proximal limb staining.

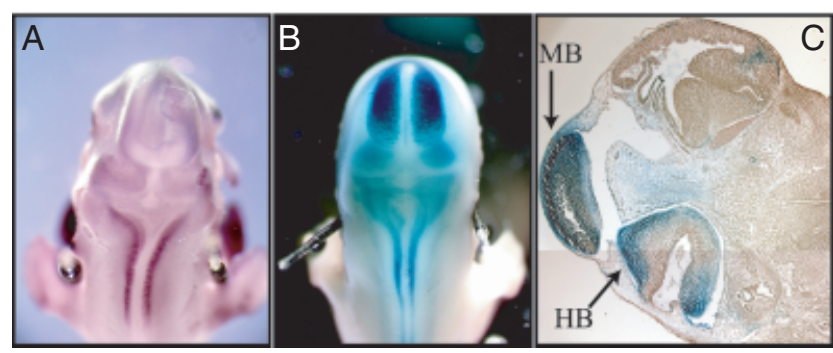

Fig. 5. Evxl versus mmA13CNS expression. (A) Evxl RNA neural tube staining at E12.5. (B) Comparable neural tube staining (lacZ) in the mmA13CNS transgenic E12.5 embryo. (C) Sixmicrometer paraffin section of the lacZ-stained mmA13CNS transgenic embryo in $\mathrm{B}$ with eosin counterstain. The hindbrain (HB) is a known site of expression for both Evxl and Evx2,

\section{DISCUSSION}

\section{A global regulatory mechanism affects not only Hoxd but also Hoxa}

Previous reports showed evidence for multiple long-range global regulatory elements $5^{\prime}$ of the HoxD cluster (Spitz et al. 2001). It has been proposed that distinct long-range enhancer elements are capable of driving neural, distal limb, and genital bud specific expression of genes within a fixed genomic region surrounding Hoxd13 from E10.5 through E13.5. The distal limb enhancer is the most finely mapped of these elements, being localized to a $54-\mathrm{kb}$ region approximately $200 \mathrm{~kb} 5^{\prime}$ of mouse Hoxd13. Spitz et al. (2003) showed that this region is capable of driving distal limb expression similar to that of the $A b d B$-like HoxD genes in two non-Hox genes: Evx2 and the newly identified Lnp gene. A $5^{\prime}$ boundary to the influence of the distal limb enhancer cannot be determined because of the dearth of genes in the region; however, this study revealed that a long-range enhancer potentially activates all genes within a finite genomic domain rather than selected promoters.

Characterizing the Hoxa13 gene neighborhood for expression during E10.5 through E13.5 revealed four consecutively arranged genes in addition to $H O X A 13$ whose expression was temporally and spatially similar, suggesting the influence of a regulatory mechanism that promotes distal limb and genital bud expression in disparate genes within a general domain. The genes within this region have an expression pattern highly similar to those under the control of the distal limb enhancer $5^{\prime}$ of the HoxD cluster. In addition, the fact that mmA13CNS can drive an Evxl-like neural expression pattern and is paralogous to mmD13CNS which is $5^{\prime}$ of Evx2 shows that the element, and probably the regulatory effect on Evx expression in the central nervous system, existed before the divergence of the Hox clusters. Altogether, it follows that the HoxA and HoxD regulatory mechanisms have been conserved since 
duplication rather than arising independently by convergent evolution.

The expression profiles of limb/genital bud expression of Hoxa13, Evx1, Hibadh, Tax1bp1, Jaz1, and Crebpa at E11.5 define a finite regulatory domain for the distal limb enhancer (Fig. 1). Based on the presence or absence of distal limb expression at E11.5, the domain in human extends across 1,227,381 bp from, but not including, $H O X A 11$ to the $5^{\prime}$ end of $C R E B 5$. This presumes that the ubiquitous expression, including distal limb and genital bud, of Jazl at this stage obscures these discrete expression domains. Based on the distal limb expression of these genes seen at E12.5 and later, it might be argued that the domain of regulation may include the promoter of $C R E B 5$. If so, the domain may be larger and lack of distal limb expression of Crebpa at E11.5 may be related to the relatively longer distance of the promoter to the regulatory element. This hypothesis might also explain the lack of tight distal autopod restriction seen with Taxlbpl and Jazl at E11.5.

Is there a role for the genes upstream of Hoxal3 to be expressed in the developing limbs and genital bud? The regulatory domains that exist upstream of the HoxA and HoxD clusters provide an intriguing problem of genome evolution in relation to long-range enhancers. Distinctly different genes occupy the regions adjacent to mmA13CNS and mmD13CNS; therefore, substantial gene insertions and/or deletions occurred near these extant conserved enhancer elements. In the context of insertions, such elements could have conferred their regulatory capabilities and restraints to the newly introduced genes. It follows that some genes expressed in new embryonic domains could be deleterious and might not be maintained. Alternatively, new gene addition(s), and expression(s), could have no effect or could lead to a selective advantage. As a result, genes having no prior role in limb or genital development could influence patterning merely by being transposed into a regulatory domain. At this time it is not known how the current genomic structure evolved or whether the genes upstream of Hoxal3 and Hoxd13 have a role in limb or genital development.

\section{Paralogous cluster noncoding sequence conservation}

It has been established that exonic/coding nucleotide sequences tend to be conserved between evolutionarily distant species, such as human and mouse, based on the functional constraints on protein coding or mRNA splicing domains. It is becoming increasingly evident that certain noncoding sequences are also similarly highly conserved. These CNSs have been associated with functional regulatory elements (Loots et al. 2000), and CNSs may act over large ( $>1 \mathrm{Mb}$ ) genomic distances (Lettice et al. 2003). Analysis of large genomic regions for potential long-range regulatory elements by com- parative sequence analysis between human and mouse homologous sequences results in a large number of CNSs. For example, 99 non-exonic CNSs greater than 100 nucleotides in length and an identity greater than $80 \%$ were found in a comparison of over $900 \mathrm{~kb} 5^{\prime}$ of $H O X D 13$ versus the homologous mouse region. Similarly, comparing over $1 \mathrm{Mb}$ of human genomic sequence $5^{\prime}$ of $H O X A 13$ with the homologous mouse sequence yielded $138 \mathrm{CNSs}$ longer than $100 \mathrm{nu}-$ cleotides with greater than $80 \%$ identity. This is not surprising given the relatively close evolutionary relationship between mouse and human. Yet, the prospect of systematically analyzing these conserved sequences for their developmental functional capacity in transgenic mice is daunting. Potential improvements in CNS selection might be made by inclusion of more distant species in the sequence analysis (Margulies et al. 2003) or by paralogous comparisons.

If the rudiments of a conserved regulatory network existed before Hox cluster divergence and remained operative, then paralogous sequence comparison should allow identification of ancient regulatory elements in common with both the HoxA and HoxD clusters that are essential for this mechanism. The fact that the $2.25-\mathrm{kb} \mathrm{mmA} 13 \mathrm{CNS}$ is so highly conserved among osteichthyans is highly suggestive of a critically important function. Because this conservation can be extended to the paralogous mmD13CNS, which is already known to be within the critical region for the HoxD distal limb enhancer, the case for mmA13CNS as a regulatory element(s) becomes stronger. Yet, the fact that this conservation is observed as far back as fugu within paraCNS4 (Fig. 2) seems counterintuitive because of the insinuated function of the distal limb enhancer in digit formation. Evidence shows that posterior HoxA orthologs in zebrafish also exhibit distal fin expression (Géraudie and Birraux 2003). Thus, the distal limb enhancer may not function per se in digit formation but merely may promote general domain and time of gene expression in appendage development, whether it is distal limb or distal fin. Alternatively, this regulatory element may have existed first to promote genital development, with involvement in limb development evolving secondarily.

A $54-\mathrm{kb}$ critical region $5^{\prime}$ of $H O X D 13$ is capable of directing distal limb mesenchymal expression of Hoxd13, and $\mathrm{mmD} 13 \mathrm{CNS}$ is located entirely within this critical region. Its paralog, mmA13CNS, by itself as a $2.25-\mathrm{kb}$ segment, does not drive reporter expression in the entire distal limb domain nor in the genital bud as expected. Yet, this sequence is sufficient for $E v x$-like reporter expression in the central nervous system. LacZ recombinant BAC constructs spanning mmA13CNS in its endogenous genomic context may help to elucidate whether additional sequences will allow for faithful distal limb and genital bud expression. If successful, an mmA13CNS-lacZdeleted BAC transgene would directly test its necessity in limb and genital bud development. 


\section{Acknowledgments}

This research was supported by the National Institutes of Health. J. A. L. and M. E. W. were supported by the University of Michigan NIH Pre-Doctoral Genetics Training Program. We thank Dr. Deneen Wellik for helpful comments on the manuscript. We thank Drs. Doug Mortlock and Ralph Dileone for technical advice regarding the Hsp68-lac Z construct. We appreciate useful comments and suggestions from our anonymous reviewers.

\section{REFERENCES}

Altschul, S. F., Gish, W., Miller, W., Myers, E. W., and Lipman, D. J. 1990. Basic local alignment search tool. J. Mol. Biol. 215: 403-410.

Bastian, H., and Gruss, P. 1990. A murine even-skipped homologue, Evx 1, is expressed during embryogenesis and neurogenesis in a biphasic manner. EMBO J. 9: 1839-1852.

Bober, E., Franz, T., Hans-Henning, A., Gruss, P., and Tremblay, P. 1994. Pax-3 is required for the development of limb muscles: a possible role for the migration of dermomyotomal muscle progenitor cells. Development 120: 603-612.

De Valck, D., et al. 1999. The zinc finger protein A20 interacts with a novel anti-apoptotic protein which is cleaved by specific caspases. Oncogene 18: 4182-4190.

DiLeone, R. J., Russell, L. B., and Kingsley, D. M. 1998. An extensive 3' regulatory region controls expression of Bmp5 in specific anatomical structures of the mouse embryo. Genetics 148: 401-408.

Dollé, P., Fraulob, V., and Duboule, D. 1994. Developmental expression of the mouse $E v x-2$ gene: relationship with the evolution of the HOM/Hox complex. Development (suppl.): 143-153.

Duboule, D., and Morata, G. 1994. Colinearity and functional hierarchy among genes of the homeotic complexes. Trends Genet. 10: 358-364.

Ferrier, D. E. K., Minguillón, C., Holland, P. W. H., and GarciaFernàndez, J. 2000. The amphioxus Hox cluster: deuterostome posterior flexibility and Hox14. Evol. Dev. 2: 284-293.

Fromental-Ramain, C., Warot, X., Messadecq, N., Lemeur, M., Dolle, P., and Chambon, P. 1996. Hoxa-13 and Hoxd-13 play a crucial role in the patterning of the limb autopod. Development 122: 2997-3011.

Géraudie, J., and Birraux, V. B. 2003. Posterior hoxa genes expression during zebrafish bony fin ray development and regeneration suggests their involvement in scleroblast differentiation. Dev. Genes Evol. 213: $182-186$.

Goodman, F. R., et al. 2000. Novel HOXA13 mutations and the phenotypic spectrum of hand-foot-genital syndrome. Am. J. Hum. Genet. 67: 197-202.

Goodman, F. R., Majewski, F., Collins, A. L., and Scrambler, P. J. 2002. A 117-kb microdeletion removing HOXD9-HOXD13 and EVX2 causes synpolydactyly. Am. J. Hum. Genet. 70: 547-555.

Hogan, B., Beddington, R., Costantini, F., and Lacy, E. 1994. Manipulating the Mouse Embryo - A Laboratory Manual. 2nd Ed. Cold Spring Harbor Laboratory Press, Plainview, NY.

Kondo, T., Zakany, J., Innis, J. W., and Duboule, D. 1997. Of fingers, toes, and penises. Nature 390: 29.
Koontz, J. I., et al. 2001. Frequent fusion of the JAZF1 and JJAZ1 genes in endometrial stromal tumors. Proc. Natl. Acad. Sci. USA 98: 6348-6353.

Kothary, R., Clapoff, S., Darling, S., Perry, M. D., Moran, L. A., and Rossant, J. 1989. Inducible expression of an hsp68-lacZ hybrid gene in transgenic mice. Development 105: 707-714.

Lettice, L. A., et al. 2003. A long-range Shh enhancer regulates expression in the developing limb and fin and is associated with preaxial polydactyly. Hum. Mol. Genet. 12: 1725-1735.

Loots, G. G., et al. 2000. Regulator of interleukins 4, 13, and 5 by crossspecies sequence comparisons. Science 288: 136-140.

Margulies, E. H., Blanchette, M., Haussler, D., and Green, E. D.; NISC Comparative Sequencing Program. 2003. Identification and characterization of multi-species conserved sequences. Genome Res. 13: 2507-2518.

McGinnis, W., and Krumlauf, R. 1992. Homeobox genes and axial patterning. Cell 68: 283-302.

Mortlock, D. P., and Innis, J. W. 1997. Mutation of HOXA13 in handfoot-genital syndrome. Nat. Genet. 15: 179-180.

Mortlock, D. P., Post, L. C., and Innis, J. W. 1996. The molecular basis of Hypodactyly $(H d)$ : a deletion in Hoxal3 leads to arrest of digital arch formation. Nat. Genet. 13: 284-289.

Post, L. C., and Innis, J. W. 1999. Altered Hox expression and increased cell death distinguish Hypodactyly from Hoxal3 null mice. Int. J. Dev. Biol. 43: 287-294.

Rougraff, P. M., Paxton, R., Kuntz, M. J., Crabb, D. W., and Harris, R. A. 1988. Purification and characterization of 3-hydroxyisobutyrate dehydrogenase from rabbit liver. J. Biol. Chem. 263: 327-331.

Ruddle, F. H., Bartels, J. L., Bentley, K. L., Kappen, C., Murtha, M. T., and Pendleton, J. W. 1994. Evolution of Hox genes. Annu. Rev. Genet. 28: 423-442.

Schwartz, S., et al. 2000. Pipmaker - a web server for aligning two genomic DNA sequences. Genome Res. 10: 577-586.

Spitz, F., Gonzalez, F., and Duboule, D. 2003. A global control region defines a chromosomal regulatory landscape containing the HoxD cluster. Cell 113: 405-417.

Spitz, F., Gonzalez, F., Peichel, C., Vogt, T. F., Duboule, D., and Zákány, J. 2001. Large scale transgenic and cluster deletion analysis of the HoxD complex separate an ancestral regulatory module from evolutionary innovations. Genes Dev. 15: 2209-2214.

Stadler, H. S., Higgins, K. M., and Capecchi, M. R. 2001. Loss of Ephreceptor expression correlates with loss of cell adhesion and chondrogenic capacity in Hoxal3 mutant limbs. Development 128: 4177-4188.

Veraksa, A., Del Campo, M., and McGinnis, W. 2000. Developmental patterning genes and their conserved functions: from model organisms to humans. Mol. Genet. Metab. 69: 85-100.

Warot, X., Fromental-Ramain, C., Fraulob, V., Chambon, P., and Dolle, P. 1997. Gene dosage-dependent effects of the Hoxa-13 and Hoxd-13 mutations on morphogenesis of the terminal parts of the digestive and urogenital tracts. Development 124: 4781-4791.

Yamamoto, M., et al. 1998. Coordinated expression of Hoxa-11 and Hoxa13 during limb muscle patterning. Development 125: 1325-1335.

Zakany, J., Fromental-Ramain, C., Warot, X., and Duboule, D. 1997. Regulation of number and size of digits by posterior Hox genes: a dosedependent mechanism with potential evolutionary implications. Proc. Natl. Acad. Sci. USA 94: 13695-13700. 